\title{
Deuteron States Model
}

\section{Gudrun Kalmbach HE*}

Mathematic informatics Naturewissenchaften, Technic, Germany

In the MINT-WIGRIS model of [1] basic states of a mathematical deuteron are described in an octonian vector space. In superposition states can be mixed like frequencies of waves. To demonstrate the inner deuteron and nucleon dynamics there are 5 videos which use the symmetries of a nucleons quark triangle, the general and special relativistic scaling factors as Moebius transformations, the projective norming of the strong interactions 5-dimensional sphere to a nucleons and atomic kernels inner spacetime as grid in its environment and Fano Gleason frames for measurements.

1.The first sequence 12 roll mill shows 6 force integrations using color charged quark nucleon triangles $r g b$ for their presentation. In between two integrations are shown the gluon exchanges between quarks.

2. The second sequence rotor presents three rotating color charged force vectors at the quark vertices in pairwise orthogonal position. This shows the confinement of quarks in a bag: an angular momentum of these rotations cannot be separated from a charged loop (the boundary of $r g b$ ), rotating in an interacting vector field which crosses the triangle area; the use of the 3-dimensional cross product is known for this as definition of induction in electromagnetism. Barycentrical coordinates are generated to determine a nucleons barycentre [2].

3. In the video membran oscillation it is shown that the vertices of the nucleon triangle are fixed in space by a rotating blue color charge momentum which lets also the gluon-sides of the triangle move between two vertices like vibrating strings. Generated are 3 cones which have the loops as boundary and the third triangle vertex as tip of the cone. For this $r g b$-gravitons are introduced.

4. The sequence pulsation shows a gravitational contraction and expansion of the nucleon triangle using these $r g b$-gravitons and phonons. The scaled 3 basic spin lengths are the triangles Bohr radii. This is an observable projection in a plane $\mathrm{E}$ of the two $r g b$ spherical nucleons from the deuteron figure. The stretching and squeezing is not in the deuteron, but only in $\mathrm{E}$ and can be seen as in a video for the actions of Moebius transformations [3] in the internet: the distance between $\mathrm{E}$ and the deuteron is changing. It is postulated that the weak and the spherical, barycentrical coordinates of the strong nucleon interaction are in special relativistic motion towards one another.

5. In the video sequence wheel two nucleon (proton, neutron) triangles are located as in the figure deuteron below. It is shown how isospin exchanges of the two nucleon proton-nucleon states generate local Euclidean spin coordinates for the weak interaction WI and electromagnetism EM.

The confinement of the quarks through the strong gluon exchange is demonstrated in the first and second video which includes the integrations for a nucleons properties and set barycenters and barycentrical coordinates. The sides of the triangle can be stretched and squeezed (video 4) in bounds given by the cones loops of video 3. The cones are whirls, not waves, with a loop oscillation added. The spherical strong coordinates are in special relativistic motion to Euclidean weak and EM coordinates generated by the wheel in video 5. General relativity as a metrical device is included in the model by its relativistic scaling factor which arises as an unsymmetric distance measure. It provides in the form of a Moebius transformation of order 6 the time cycles of the strong rotor and weak wheel.

The Fano figure for octonians (see for more MINT-WIGRIS figures in the internet) allow seven measuring triples like the Pauli spin 123 spin coordinate and Pauli matrices for space (at 1 red in the cube). Deleting the letter $e$ in the octonian coordinate list, the octonian triples in the Fano figure are 123, 145, 167, 246, 257, 347, 356. The space 356 is for the SI rotor with barycentrical coordinates of the triangle. 167 is for periodic functions and light with cylindrical coordinates (at 7). Other GF lines are 145 (at 4, cone, magnetism, color charge y), 246 (at 2, sphere $\mathrm{S}^{2}$, entropy, color charge g), 257 (at 5, barycenter, mass and gravity, color charge t), 347 (at 3 , rotation axis with an orthogonal orbit plane, wheel, color charge magenta), 356 (at 6, kinetic frequency, rotor, color charge $b$. The triples are taken as 3-dimensional frames for Gleason measures. They arise through the real cross product, known for the Pauli spin matrix multiplication. It is not commutative like most operator compositions. In chapter 6 from them are constructed real Gleason measures which can extend to complex measures on the complex 3-dimensional space $C^{3}[1]$. They allow three real weights for their coordinates. If the complex version is drawn as the vectorial caps on the hedgehog sphere $S^{2}$ the weights can be extended to 6 . This is also the order of the matrix $G$ arsing from the general relativistic scaling factor related to the Schwarzschild radius Rs. As sixth roots of unity the action of $G$ is like a discrete compass whose needle can set in $\pi / 3$ rotation six numerical weights, but it also sets color charges on the circular segments between them or 6 electrical charges. The $r g b$ graviton whirl figure shows 3 such segments for the quark triangle whirls in a nucleon. The whirls stack like magnetic field quantum whirls (used for developing quantum computers). The rgb frame 126 is a cross product of the Gell-Mann matrices $\lambda 1, \lambda 2, \lambda 3$. As nucleons neutral color charge of quarks 126 allows the SI rotor whirl rotation for integrations (Figure 1).

\section{Deuteron}

quarks $1 \mathrm{u}, 2 \mathrm{u}, 6 \mathrm{~d}, 3 \mathrm{~d}, 4 \mathrm{u}, 5 \mathrm{~d}$, gluon exchanges 12,16,26,34,35,45, weak isospin exchanges $15,23,46,126,345$ nucleon $r g b$-graviton locations, observed stretching/squeezing through a central projection [4], 6 roll mill driven by 3 motors [5,6] gravity plus electrical potentials

*Corresponding author: Gudrun Kalmbach Mathematics Professor, Inventor of MINT (Mathematic informatics Naturewissenchaften, Technic), Germany, Tel: +49 (0) 30 25418-499; E-mail: mint-01@maxi-dsl.de

Received December 03, 2017; Accepted December 25, 2017; Published January 10, 2018

Citation: Gudrun Kalmbach HE (2018) Deuteron States Model. J Laser Opt Photonics 5: 172. doi: 10.4172/2469-410X.1000172

Copyright: (c) 2018 Gudrun Kalmbach HE. This is an open-access article distributed under the terms of the Creative Commons Attribution License, which permits unrestricted use, distribution, and reproduction in any medium, provided the original author and source are credited. 


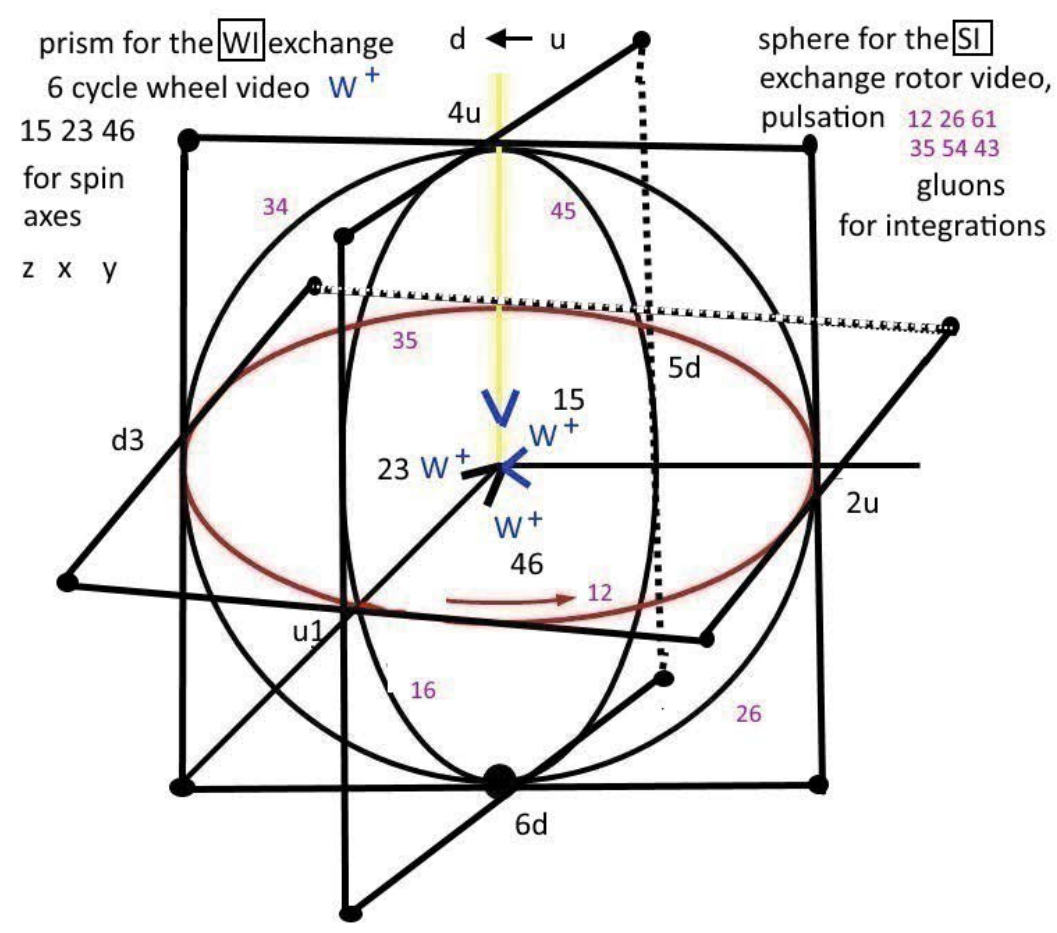

Figure 1: Deuteron.

$[3]^{1}$ POTm (rolls color charges red rebar as Heisenberg uncertainties couplings), strong motor SIm (rolls green greenbar) and weak motor WIm (rolls blue yellow).

${ }^{1} \mathrm{~A}$ comment is made to the article [5], concerning the escape (second cosmic) speed (p. 11) $R s / r=v 2^{2} / c^{2}$ : if two systems are not in gravitational interaction, for the Minkowski metric the escape speed is used and the general relativistic (radius distance) scaling factor as $\sin ^{2} \beta=R s / r$ is replaced by the special relativistic (speed distance) scaling factor as $\sin ^{2} \delta=\mathrm{v}^{2} / \mathrm{c}^{2}$. - Concerning the projective norming of the 5-dimensional spheres boundary $S^{2}$ : the extended linear dependent $\sigma 3$ Pauli matrices in $\mathrm{SU}(3)$ generate a projection plane $E$ from $\infty$ in $S^{2}$ onto $E$ for (distance [6]) stretching/squeezing

\section{References}

1. G Kalmbach HE (2017) MINT-WIGRIS, MINT Verlag, Bad Woerishofen.

2. G Kalmbach HE (1997-2017) (Chef-Hrsg.), MINT (Mathematik, Informatik, Naturwissenschaften, Technik), MINT Verlag, Bad Woerishofen, Vol: 1-36.

3. Poston T and Stewart I (1978) Catastrophe theory and its applications, Pitman, London.

4. Schmutzer E (2004) Projektive einheitliche Feldtheorie, Harry Deutsch, Frankfurt.

5. G Kalmbach HE (2017) Deuteron States ${ }^{1}$. Nessa J Phys 1: 1-17.

6. (2014) Internet video under YouTube: Moebius Transformations Revealed. 